University of New Hampshire

University of New Hampshire Scholars' Repository

Space Science Center

Institute for the Study of Earth, Oceans, and

Space (EOS)

1991

\title{
COMPTEL images locations of gamma-ray bursts
}

\author{
$M$ Varendorff \\ Max-Planck-Institut für extraterrestrische Physik \\ K Bennett \\ ESTEC \\ $\mathrm{H}$ deBoer \\ SRON \\ M Busetta \\ ESTEC \\ H Bloemen \\ Space Research Organization of the Netherlands
}

See next page for additional authors

Follow this and additional works at: https://scholars.unh.edu/ssc

Part of the Astrophysics and Astronomy Commons

\section{Recommended Citation}

COMPTEL images locations of gamma-ray bursts Varendorff, M. G. and Bennett, K. and de Boer, H. and Busetta, M. and Bloemen, H. and Collmar, W. and Connors, A. and Diehl, R. and den Herder, J. W. and Hermsen, W. and Kippen, M. and Kuiper, L. and Lichti, G. and Lockwood, J. and McConnell, M. and Morris, D. and Much, R. and Ryan, J. and Schönfelder, V. and Stacy, G. and Steinle, H. and Strong, A. W. and Swanenburg, B. N. and de Vries, C. and Winkler, C., AIP Conference Proceedings, 265, 77-81 (1991), DOI:http://dx.doi.org/10.1063/1.42782

This Conference Proceeding is brought to you for free and open access by the Institute for the Study of Earth, Oceans, and Space (EOS) at University of New Hampshire Scholars' Repository. It has been accepted for inclusion in Space Science Center by an authorized administrator of University of New Hampshire Scholars' Repository. For more information, please contact Scholarly.Communication@unh.edu. 


\section{Authors}

M Varendorff, K Bennett, H deBoer, M Busetta, H Bloemen, W Collmar, A Connors, R Diehl, J W. den Herder, W Hermsen, R M. Kippen, L Kuiper, G G. Lichti, J Lockwood, Mark L. McConnell, D Morris, R Much, James M. Ryan, V Schonfelder, G Stacy, H Steinle, A W. Strong, B Swanenburg, C de Vries, and C Winkler 


\section{AIP | Proceedings}

\section{COMPTEL images locations of gammaray bursts}

M. G. Varendorff, K. Bennett, H. de Boer, M. Busetta, H. Bloemen, W. Collmar, A. Connors, R. Diehl, J. W. den Herder, W. Hermsen, M. Kippen, L. Kuiper, G. Lichti, J. Lockwood, M. McConnell, D. Morris, R. Much, J. Ryan, V. Schönfelder, G. Stacy , H. Steinle, A. W. Strong, B. N. Swanenburg, C. de Vries, and C. Winkler

Citation: AIP Conference Proceedings 265, 77 (1991); doi: 10.1063/1.42782

View online: http://dx.doi.org/10.1063/1.42782

View Table of Contents:

http://scitation.aip.org/content/aip/proceeding/aipcp/265?ver=pdfcov

Published by the AIP Publishing

Articles you may be interested in

Spectral evolution of gammaray bursts

AIP Conf. Proc. 265, 169 (1991); 10.1063/1.42820

BATSE observations of gammaray bursts

AIP Conf. Proc. 265, 13 (1991); 10.1063/1.42813

OSSE observations of cosmic gammaray bursts AIP Conf. Proc. 265, 32 (1991); 10.1063/1.42804

Gamma ray burst source locations with the Ulysses/Compton/PVO Network AIP Conf. Proc. 265, 72 (1991); 10.1063/1.42781

Gammaray bursts observed by the watch experiment AIP Conf. Proc. 265, 53 (1991); 10.1063/1.42778 


\section{COMPTEL IMAGES LOCATIONS OF GAMMA-RAY BURSTS}

M. G. Varendorff ${ }^{1}$, K. Bennett ${ }^{4}$, H. de Boer ${ }^{2}$, M. Busetta ${ }^{4}$, H. Bloemen ${ }^{2}$, W. Collmar ${ }^{1}$, A. Connors ${ }^{3}$, R. Diehl ${ }^{1}$, J. W. den Herder ${ }^{2}$, W. Hermsen ${ }^{2}$, M. Kippen ${ }^{3}$, L. Kuiper ${ }^{2}$, G. Lichti ${ }^{1}$, J. Lockwood ${ }^{3}$, M. McConnell ${ }^{3}$, D. Morris $^{3}$, R. Much ${ }^{1}$, J. Ryan ${ }^{3}$, V. Schönfelder ${ }^{1}$, G. Stacy ${ }^{3}$, H. Steinle ${ }^{1}$, A. W. Strong ${ }^{1}$, B. N. Swanenburg ${ }^{2}$, C. de Vries ${ }^{2}$, C. Winkler ${ }^{4}$

${ }^{1}$ Max-Planck Institut für extraterrestrische Physik, 8046 Garching, F.R.G.

${ }^{2}$ Laboratory for Space Research Leiden, 2300 RA Leiden, The Netherlands

${ }^{3}$ University of New Hampshire, Durham, NH 03824, U.S.A.

${ }^{4}$ Space Science Department of ESA, ESTEC, 2200 AG Noordwijk, The Netherlands

\section{ABSTRACT}

The $\gamma$-ray telescope COMPTEL onboard GRO has so far located 6 gammaray bursts which occured in its $\sim 1 s r$ field of view. The positions of the sources were derived by the maximum-entropy method. Systematic and statistical uncertainties for the four strongest bursts are approximately $1^{\circ}$ to $2^{\circ}$ and can be reduced in future analysis.

\section{INTRODUCTION}

The imaging $\gamma$-ray telescope COMPTEL is one of four instruments on board NASA's Gamma Ray Observatory (GRO), which was launched by the space shuttle Atlantis on April 5, 1991. In the first year of the mission COMPTEL will perform a survey of the whole sky in the energy range from $1 \mathrm{MeV}$ to $30 \mathrm{MeV}$ with an energy resolution of $5 \%$ to $10 \%$ FWHM and an angular resolution of about $1^{\circ}$ to $2^{\circ}(1 \sigma)$, depending on the gamma-ray energy. A description of COMPTEL's burst detection capabilities together with results from GRB910503 is given by Winkler et al. ${ }^{1}$ A detailed description of the instrument and its technical properties is given by Schönfelder et al. ${ }^{2}$ COMPTEL has two different capabilities for burst measurements. First, the telescope itself for imaging of bursts in the $1 \mathrm{sr}$ field of view and second two quasi omni-directional burst detectors for derivation of spectra from all detected bursts. The burst trigger function is performed by BATSE for all GRO instruments.

During the first months of the mission, COMPTEL has detected many gamma-ray bursts. Most of them are not in the field of view, so only spectral information in the energy range $0.1 \mathrm{MeV}$ to $10 \mathrm{MeV}$ is available. For those, in its field of view, COMPTEL can provide images in the energy range from about 1 to $30 \mathrm{MeV}$. This is the first time that positions of gamma-ray bursts at $\mathrm{MeV}$ energies are determined by one single instrument. In this paper we report on the first results of the stronger bursts in the field of view.

\section{ANALYSIS METHOD}

With present Compton telescopes no simple imaging of the sky is possible. The source distribution in the sky has to be derived by combining the 
information provided by the measured events together with the response of the instrument. At present, three different methods are used by the COMPTEL team. These are the maximum-likelihood method, the angular resolution measure (ARM) method, and the maximum-entropy method. The likelihood method searches for a best fit of a model sky to the data, after convolution with the insrumental response. The ARM-method does not require detailed knowledge of the response; the distribution of events in a one-dimensional projection of the data space is determined (the so called ARM-distribution ${ }^{3}$ ) for different trial source positions. A peak near the zero-point of this distribution with the angular width of the point spread function indicates the presence of a source at the trial position.

In this paper results of the maximum-entropy method are presented. For this analysis the software package MEMSYS 2 from Maximum-Entropy Data Consultants Ltd. was used. A detailed explanation of the method is given by Skilling and Bryan. ${ }^{4}$ The application to this Compton telescope is discussed by Strong et al..$^{5}$ Only the main characteristics of this method are described here. A distribution of sources on an 'input' sky is searched, which matches the data after convolution with the point spread function and fullfills the maximumentropy criterion. This criterion determines the smoothest 'input' sky which deviates, after convolution, not more than one standard deviation from the data. This method requires a detailed knowledge of the point spread function of the instrument. The telescope response to a gamma-ray point source can be described in the simplest way in a three-dimensional data space, defined by the scatter direction $(\chi, \psi)$ and the Compton scatter angle $\bar{\varphi}$ (see Figure 1).

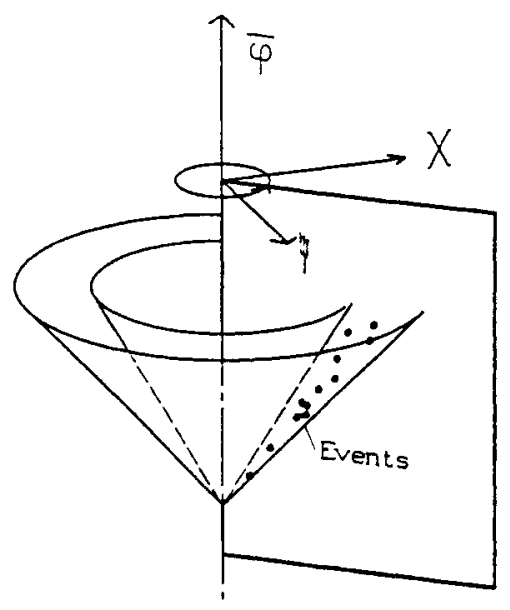

Figure 1. Three-dimensional data space.

Each detected photon is represented by one point in this three-dimensional data space. In the idealised case, in which the scattered gamma-ray is totally absorbed in the lower detector, the pattern of all data points resulting from a point source with coordinates $\left(\chi_{0}, \psi_{0}\right)$ lies on a cone in the $(\chi, \psi, \bar{\varphi})$-space, where the cone apex is at $\left(\chi_{0}, \psi_{\circ}\right)$, and the cone semi-angle is $45^{\circ}$. For real measurements, this cone mantle is blurred due to measurement uncertainties and especially due to incomplete absorption in the lower detector. 


\section{RESULTS}

Up to now 6 gamma-ray bursts in the field of view have been analyzed. A list of these bursts including onset times and preliminary locations are given in Table I. Four of these bursts (GRB 910425, GRB 910503, GRB 910601 and GRB 910814) are strong events with about 100 counts. The time profile of GRB 910503 is given by Winkler et al. ${ }^{1}$ The time profiles of GRB 910425, GRB 910601 and GRB 910814 are shown in Figure 2.

These bursts have also been observed by ULYSSES, enabling the determination of 'triangulation circles.' COMPTEL images together with these circles are shown in Figure 3. An image of GRB 910503 is given by Winkler et al. ${ }^{1}$ At the moment, both the statistical and the systematic uncertainties in the positions of the four strongest bursts are about $1^{\circ}$ to $2^{\circ}$. The statistical errors for the positions of the two weaker bursts are not determined, but are evidently larger than for the stronger bursts.
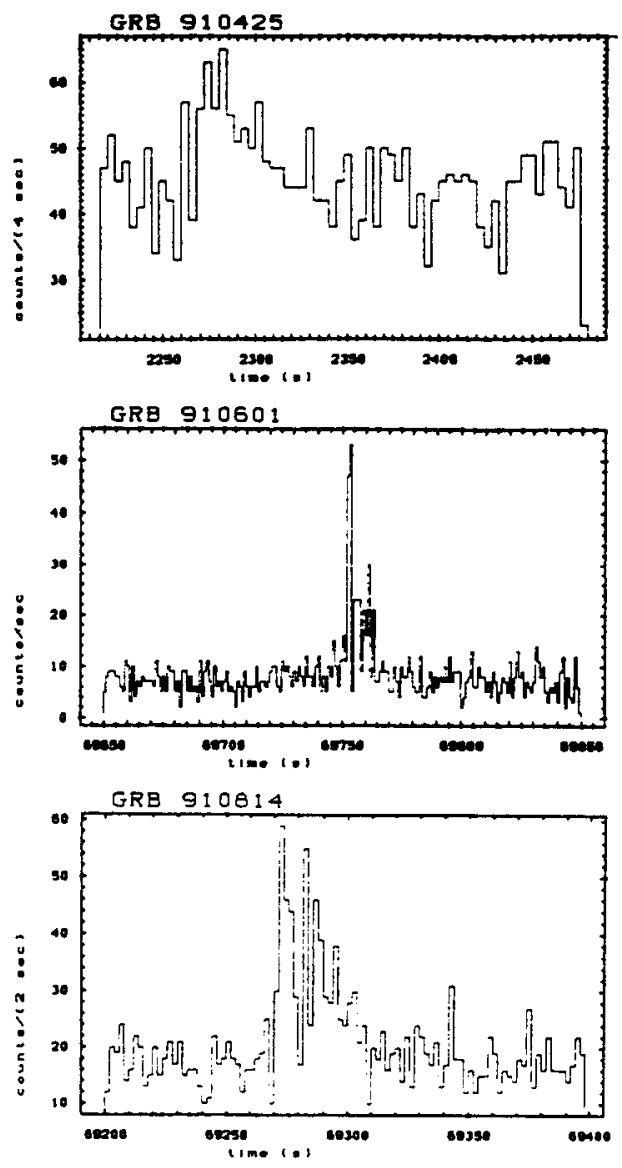

Figure 2. Time profiles of GRB 910425, GRB 910601 and GRB 910814. 

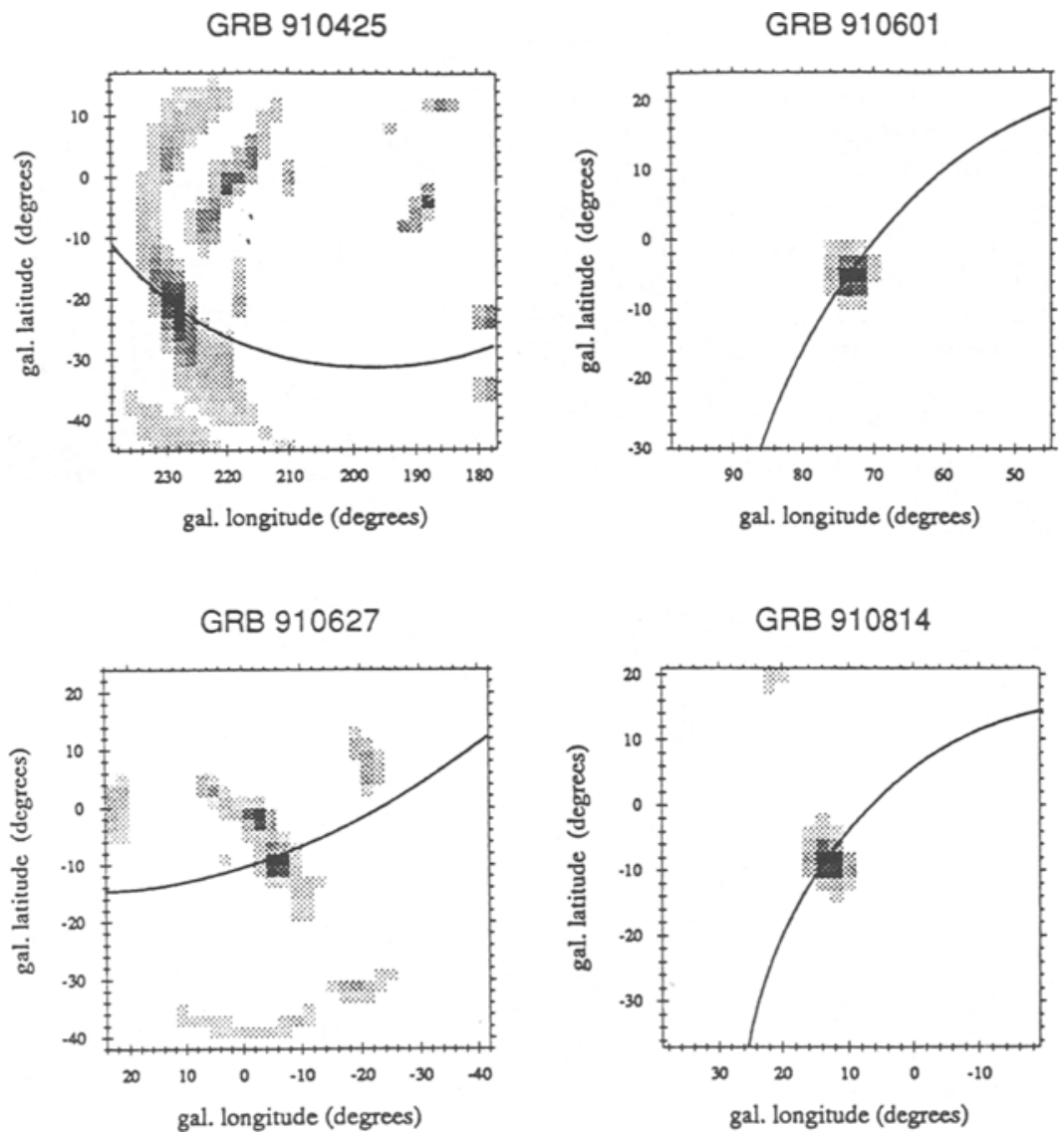

Figure 3. Maximum-entropy images of GRB 910425, GRB 910601, GRB 910627, and GRB 910814.

The other two gamma-ray bursts (GRB 910609 and GRB 910627) are weaker events with $\leq 30$ counts in the data. The statistical error of the position of these bursts is not yet estimated, but according to the smaller number of counts, it is larger than for the stronger bursts. The first burst GRB 910609 has only 6 counts, but all 6 events fell into a $14 \mathrm{~ms}$ time interval. The rate of background at this time is at about one event per second. According to BATSE measurements (private communication), this burst is $63^{\circ}$ away from the zenith of the instrument and is a strong event, which extends into the $\mathrm{MeV}$-range. The nominal field of view of COMPTEL has a radius of $\sim 30^{\circ}$ around the zenith. At the position of $63^{\circ}$ from zenith the sensitivity of the telescope is very low and the standard response is not applicable to the data. As a result the position derived has a large systematic error $\geq 10^{\circ}$.

The second weak burst GRB 910627 was also observed by Ulysses and the 
COMPTEL image (Figure 3 ) is in good agreement with the triangulation circle, although the image is based on only 30 events.

\begin{tabular}{|cc|ccc|c|}
\hline \multicolumn{4}{|c|}{ Table I. Burst onset times and locations. } \\
\hline Date & Seconds & gal. Longitude & gal. Latitude & Triangulation \\
\hline 910425 & 2268 & 229. & -20. & yes \\
910503 & 25455 & 171. & 7. & yes \\
910601 & 69736 & 72.5 & -5. & yes \\
$910609^{*}$ & 2909 & 240. & -10. & no \\
910627 & 16159 & 318. & 59. & yes \\
910814 & 69275 & 95. & -28. & yes \\
\hline
\end{tabular}

* big systematic error in position.

\section{SUMMARY}

While COMPTEL images per se do not significantly improve the chances of finding counterparts, in combination with Ulysses or PVO timing data 'finding charts' are developed with small image dimensions (e.g., $1^{\circ} \times 1^{\prime}$ for GRB 910503). This is a powerful tool in the search for suitable counterparts which may lead eventually to solving the GRB mystery.

\section{REFERENCES}

1. C. Winkler et al., these proceedings.

2. V. Schönfelder et al., IEEE Trans. Nucl. Sci. NS - 31, 766 (1984).

3. J. W. den Herder et al., "Comptel: instrument description and performance," presented at the Second GRO Science Workshop, Annapolis, MD (1991).

4. J. Skilling and R. K. Bryan, "Maximum-entropy image reconstruction: general algorithm," MNRAS 211, 111 (1984).

5. A. W. Strong et al., Proc. XXI Int1. Cosmic Ray Conf., Adelaide 4, 154 (1990).

\section{ACKNOWLEDGEMENT}

We are grateful to the Ulysses team for providing us the Ulysses arrival times of GRB 910425, GRB 910503, GRB 910601, GRB 910627 and GRB 910814. Furthermore we thank the BATSE team for the burst triggers. 